\title{
ANÁLISIS DEL DISCURSO APLICADO AL APRENDIZAJE DE COMPETENCIAS COMUNICATIVAS EN EL DOMINIO DEL TURISMO
}

\section{DISCUSSION ANALYSIS APPLIED TO THE LEARNING OF COMMUNICATIVE SKILLS IN THE FIELD OF TOURISM}

\author{
Olivier Gérard Angel Méric, PhD. \\ Doctor en letra moderna (Francia). \\ Docente investigador de la Universidad Estatal Amazónica, Ecuador. \\ olivier.meric.a@gmail.com
}

\section{ARTÍCULO DE REFLEXIÓN}

Recibido: 27 de junio de 2017

Aceptado: 13 de agosto de 2017

\section{RESUMEN}

El artículo se enfoca en las posibles aplicaciones didácticas de los resultados obtenidos a partir del análisis del discurso. Luego de definir el marco teórico del discurso especializado, se establece que el discurso profesional tiene las mismas características que el discurso "común" y así determinar que no es una necesidad adquirir conocimiento lingüístico general previo al aprendizaje del idioma con fines específicos. El artículo sugiere que la lingüística de corpus puede ayudar en diseñar los contenidos del proceso de enseñanza-aprendizaje de un idioma extranjero lo que se ilustra con el análisis pragmático de los componentes del corpus compilado a partir de las "anécdotas". Este corpus ha sido elaborado para el proyecto europeo lingüístico KA2 cuyo nombre es "Tell me A Story".

Palabras clave: lingüística de corpus, análisis del discurso, didáctica, discurso especializado 


\section{ABSTRACT}

This paper focuses on possible didactic applications of corpus linguistic analysis results. After having stated the theoretical frame around the concept of specialized discourses within a professional context, it is firstly argued that professional discourses use the same linguistic patterns than « general » discourse; hence, it is not necessary to acquire previous general knowledge before learning language for specific purposes (LSP). Secondly, this paper suggests that corpus linguistic can help to design the contents used for teaching communicative competences what is illustrated through the analysis of the pragmatic patterns present in a corpus made of "short stories". This corpus has been set for a European KA2 language project named "Tell Me A Story".

Keywords: corpus linguistics, discourse analysis, didactics, specialized discourse,

\section{INTRODUCCIÓN}

El manejo de las competencias comunicativas es una condición necesaria a la integración social del individuo desenvolviéndose en un mundo tan conectado como el nuestro. La omnipresencia de la conectividad en todos los dominios - que sean educacionales, profesionales, públicos o personales - es una de las razones por las cuales se aprenden diferentes herramientas de comunicaciones. En el dominio profesional, y aún más en el dominio del turismo, los expertos admiten que el manejo de técnicas de comunicaciones en varios idiomas extranjeros es una competencia indispensable al desempeño profesional, razón por la cual este aprendizaje tiene una atención particular en las mallas curriculares de las carreras en turismo. La lectura de la obra de Germain ofrece una documentación detallada de las principales escuelas de la didáctica de las lenguas; en relación al modelo de Legendre expuesto por Germain (1993, pp. 12-14), este trabajo no pretende exponer una variante metodológica, pero tomando en cuenta el principio de que la elección de lo que se debe enseñar tiene un impacto directo sobre la manera de enseñarlo (Richard, 2001), el estudio presentado se enmarca en la relación didáctica que existe entre el objeto y el actor profesional. Teniendo en cuenta los objetivos específicos de la formación de los estudiantes quienes desean ser profesionales en el dominio del turismo, este artículo 


\section{Revista Científica ECOCIENCIA}

propone una discusión analítica de un género de discurso profesional especializado en turismo, la anécdota, como recurso de aprendizaje para definir la selección de contenidos apropiados a la adquisición de las "competencias comunicativas", tal como las define Hymes (1992, pp. 51-52).

En primer lugar, las diferencias entre discurso especializado y discurso de especialidad serán expuestas con el afán de ubicar el marco teórico en el cual la anécdota será considerada. Luego, una definición más detallada del concepto de anécdota será propuesta tal como fue aplicada en la realización del proyecto "Tell me a story". En una tercera parte, se presentará un enfoque pragmático del análisis del corpus compilado, antes de concluir sobre las posibles implicaciones didácticas de los resultados obtenidos con el fin de valorar sus contribuciones en el concepto pedagógico del proceso de enseñanza-aprendizaje de las competencias comunicativas.

\section{REVISIÓN TEÓRICA}

\section{Distinción entre «discurso de especialidad» $y$ «discurso especializado»}

En el marco de un programa enfocado sobre objetivos específicos, el contenido a trasmitir determina las elecciones didácticas; es entonces importante definir la esencia misma de lo que se enseñará. En la norma ISO 1087, en 1990, se propone definir la lengua de especialidad como un: "subsistema que emplea una terminología y otros medios lingüísticos apuntando a la non-ambigüedad de la comunicación en un dominio particular" (ISO 1087, 1990). Aparece un concepto de idiomas asociado a tal o cual dominio de actividad, presentando la estructura de un "sub-sistema" sin aportar una definición del sistema de referencia, y sin indicar en que estos sub-sistemas se diferencian los unos de los otros. El documento infiere la existencia de un sistema sin referirse a otra norma que pudiera dar una descripción invocando algunos medios lingüísticos asociados o no a la terminología para la descripción de estos sub-sistemas que apuntan a la non-ambigüedad de la

\footnotetext{
${ }^{1}$ Salvo indicaciones contrarias, las traducciones al español son propuesta por el autor y la versión original se consignará en nota de pie de página: "sous-système qui utilise une terminologie et d'autre moyens linguistiques et qui vise la non-ambigüité de la communication dans un domaine particulier»
} 
comunicación, lo cual es sumamente ambiguo. Aún si esta norma fue revisada en dos ocasiones, esta falta de precisión se traduce, al nivel didáctico, por el uso de la lengua, descrita como un sistema, y de su terminología asociada para caracterizar el dominio de especialidad. Entonces, el discurso de especialidad es a menudo reducido al estudio de un catálogo de situaciones enfocándose en expresiones estereotipadas. Pierre Lerat (1995, p. 20) propuso que el lenguaje especializado sea definido como "un lenguaje natural vector de conocimiento especializado"".

La supresión del concepto sistémico y estructural marca un cambio de paradigma (Gautier, 2014). En este enfoque, la especialidad ya no se aprehende según su terminología sino según su realidad pragmática. Los contenidos de aprendizaje emanan de un análisis del discurso producido en situación de comunicación propia a la especialidad. Esta definición asocia el lenguaje de especialidad a conocimientos de especialidad a pesar de no mencionar las competencias. En la definición de Michel Petit la noción del lenguaje de especialidad desaparece totalmente al beneficio del lenguaje especializado que se posiciona como un elemento constitutivo del dominio especializado, el cual se vuelve la entidad de referencia:

Llamaremos dominio especializado todo sector de la sociedad constituido alrededor y por el ejercicio de una actividad que, por su naturaleza, su finalidad, sus modalidades específicas y por las competencias específicas que necesitan sus actores, define el lugar fácilmente identificable del sector dentro de la sociedad y el lugar de un conjunto sectores determinando su composición y su organización específica. $^{3}$ (Petit, 2010, p. 9)

Michel Petit presenta el dominio especializado como una actividad que requiere el manejo de competencias particulares entre las cuales las competencias relacionadas a la comunicación que el mismo clasifica en tres formas discursivas: el especializado académico (investigación en el dominio); el especializado profesional (actividad propia al dominio); y el

\footnotetext{
2 «une langue naturelle considérée en tant que vecteur de connaissances spécialisées »

3 « Nous appellerons domaine spécialisé tout secteur de la société constitué autour et en vue de l'exercice d'une activité principale qui, par sa nature, sa finalité et ses modalités particulières ainsi que par les compétences particulières qu'elle met en jeu chez ses acteurs, définit la place reconnaissable de ce secteur au sein de la société et d'un ensemble de ses autres secteurs et détermine sa composition et son organisation spécifiques. »
} 


\section{Revista Científica ECOCIENCIA}

especializado "del tercer tipo" (el discurso sobre el dominio, pero fuera de su actividad propia). Este enfoque pragmático, que centra el aprendizaje en el dominio y no en la existencia supuesta de un sub-sistema lingüístico, vuelve obsoleto el debate de sobre la necesidad de exponer el estudiante al idioma natural antes de planificar situaciones comunicacionales directamente relacionadas al dominio (Lavric, 2013). En el entorno de un curso de idioma extranjero propuesto a estudiantes de nivel A1, definido por el Marco Europeo Común de Referencia para la Enseñanza de los Idiomas (Conseil de l'Europe, 2001, p. 23), dentro de una formación en turismo, ¿existe un interés particular enseñar "es un deportista" (Girardet \& Pecheur, 2006, p. 11) en lugar de "es un turista"? Según Richard (2001, p. 51), una de las consideraciones básicas de la elaboración de un programa educativo es que debe ser centrada en el análisis de las necesidades de los estudiantes, aún más en discurso especializado y en los programas de formación profesional.

El análisis de las necesidades y de los objetivos del público estudiantil condiciona la composición de los contenidos del nivel A1 hasta el nivel C2. En el dominio del turismo, los editores de recursos didácticos proponen libros de aprendizaje que se adaptan a un público cada vez más principiante. Los libros de Corbeau, Dubois \& Penfomis (2004) y Corbeau, Dubois, Penfomis \& Semichon (2013) sugieren sesenta horas de enseñanza del francés "ordinario" antes de utilizar sus obras. El docente no tiene la obligación de introducir sistemáticamente el dominio en los contenidos del idioma estudiado, pero puede orientar su proceso de enseñanza / aprendizaje hacia el discurso especializado de las situaciones de comunicación del dominio. Puede elegir el proceso de un aprendizaje pragmático y contextualizado de un idioma "situado" en lugar de un proceso centrado sobre la existencia supuesta de un sub-sistema lingüístico de tal o cual dominio especializado.

\section{La anécdota como contenido de arranque del aprendizaje}

Pragmáticamente, el contenido que el docente propone a sus estudiantes puede ser definido gracias a las metodologías del análisis del discurso aplicado al dominio estudiado. Según la definición propuesta por Gunnarsson (2009, p. 5), el discurso profesional está constituido de textos involucrando por lo menos un profesional dentro de un contexto con fines específicos. Estos textos, compilados en un corpus, pueden ser sometidos a un 
análisis cuantitativo con la ayuda de herramientas informáticas cuya interpretación cualitativa tiene como objetivo la identificación de las "modalidades particulares" (Petit, 2010, p. 9) y de las características del dominio.

En relación al turismo, la diversidad textual, en parte debida a los diferentes medios de difusión, llevó los investigadores como Calvi (2010) o Mapelli \& Piccioni (2011) a identificar varios géneros discursivos con características propias a las interacciones producidas en las diferentes situación comunicativas donde se entremezclan, a diferentes niveles, las tres competencias comunicativas: lo lingüístico, lo cognitivo y lo pragmático. La lingüística basada en el estudio de corpus permite la extracción de ciertas características de los componentes genéricos del molde discursivo con miras a una aplicación didáctica. En este trabajo, el tipo de texto elegido, la anécdota, se enmarca dentro de los textos emotivos y conativos a disposición del guía turístico en situación profesional cuando se dirige a un grupo de visitantes. Según Lionel Gossman (2003), el término "anécdota" queda mal definido, aún si le asocia a una narración diminuta y confidencial centrada en una estructura tripartita: la descripción del contexto, la intriga y el desenlace con su "lección".

Esta concepción concuerda con la definición de la real academia: "del fr. anecdote, y este del gr. ávéкঠота anékdota 'cosas inéditas'. Relato breve de un hecho curioso que se hace como ilustración, ejemplo o entretenimiento." Se encuentra el componente emotivo en la palabra "entretenimiento" y el conativo en la "ilustración" o en el "ejemplo", pero es por el "hecho curioso" que este género discursivo fue seleccionado para la realización del proyecto europeo "Tell Me A Story" (TMAS) 4 financiado por la comisión europea para la educación continua. Uno de los objetivos del proyecto, que incluye ocho idiomas europeos, era de utilizar las anécdotas en relación con ocho ciudades europeas o regiones europeas para contarlas en una visita turística y suscitar el interés de los guías profesionales para la adquisición de una nueva lengua extranjera. Este género textual fue elegido por su capacidad en despertar la curiosidad de los visitantes amantes de detalles a menudos en margen de la información oficial.

\footnotetext{
${ }^{4}$ Lifelong Learning Programme, Tell Me A Story, http://tellmeastory.eu/. Ref. No.: 531009-LLP-2012-BG-KA2-KA2MP.
} 


\section{Corpus y análisis}

\section{Constitución del corpus}

Una vez definidos los elementos constitutivos del corpus, fue decidido integrar aleatoriamente una parte de las anécdotas publicadas en las páginas del sitio internet de la revista "La France Pittoresque". El corpus obtenido totaliza 944.877 tokens distribuidos en seis temáticas nombradas por el comité editorial de la revista: "insólitos, cortos, costumbres y tradiciones, eventos, fauna y flora, e históricos ${ }^{5}$. Los temas abordados en estas anécdotas son tantos variados y extendidos como los temas encontrados en el mundo profesional de las visitas turísticas. Las características de constitución del corpus permiten analizar un número de texto suficientemente importante para representar el género discursivo de la anécdota tal como fue definido anteriormente insertándose en el macrogénero de la "guia turística" incluida en la familia de género "institucional" según la taxonomía propuesta por Mapelli \& Piccioni (2011) en relación al turismo.

\section{$\underline{\text { Análisis }}$}

La Tabla 1 presenta los resultados del análisis comparativo entre el corpus compilado por las necesidades de la presente investigación y el corpus frTenTen12, considerado como representativo de un uso general del idioma francés por los 12 miliares de palabras distribuidas en más de $\mathbf{4 8 0 . 0 0 0 ~ o r a c i o n e s ~ t i p o g r a ́ f i c a s . ~ L a s ~ e s t a d i ́ s t i c a s ~ p r e s e n t a d a s ~ f u e r o n ~}$ obtenidas gracias al programa "Sketch Engine" (Kilgarriff, 2012).

Tabla 1. Comparación de las características, frecuencias y porcentajes de la naturaleza de las palabras constitutivas de los corpus «anécdota»y «frTenTen12».

\begin{tabular}{|c|c|c|c|}
\hline \multicolumn{2}{|c|}{ Corpus "anécdota" } & \multicolumn{2}{|c|}{ Corpus “FrTenTen12” } \\
\hline Unit & Frequency & Unit & Frequency \\
\hline Tokens & 1078496 & Tokens & 12369868562 \\
\hline Sentences & 30647 & Sentences & 481519836 \\
\hline
\end{tabular}

\footnotetext{
5 «insolites, brèves, coutumes et traditions, événements, faune et flore et historiques ».
} 


\begin{tabular}{|c|c|c|c|c|c|}
\hline Part of speech & Freq. & $\%$ & Part of speech & Freq. & $\%$ \\
\hline noun & 203118 & 18,83 & noun & 2495153630 & 20,17 \\
\hline preposition & 126961 & 11,77 & preposition & 1377841597 & 11,14 \\
\hline article & 103571 & 9,60 & article & 1010825843 & 8,17 \\
\hline punctuation & 94455 & 8,76 & punctuation & 869394390 & 7,03 \\
\hline personal pronoun & 58443 & 5,42 & personal pronoun & 571659495 & 4,62 \\
\hline adverb & 56671 & 5,25 & adverb & 647997257 & 5,24 \\
\hline adjective & 56242 & 5,21 & adjective & 855755229 & 6,92 \\
\hline conjunction & 49607 & 4,60 & conjunction & 512463124 & 4,14 \\
\hline verb present & 43821 & 3,20 & verb present & 679293706 & 5,49 \\
\hline sentence tag & 34512 & 3,20 & sentence tag & 456741634 & 3,69 \\
\hline proper name & 31546 & 2,92 & proper name & 537729360 & 4,35 \\
\hline verb past participle & 30126 & 2,79 & verb past participle & 334596987 & 2,70 \\
\hline verb infinitive & 25708 & 2,38 & verb infinitive & 347805390 & 2,81 \\
\hline relative pronoun & 20019 & 1,86 & relative pronoun & 149125717 & 1,21 \\
\hline verb imperfect & 18499 & 1,72 & verb imperfect & 99097964 & 0,80 \\
\hline demonstrative pronoun & 17227 & 1,60 & demonstrative pronoun & 170454362 & 1,38 \\
\hline numeral & 16834 & 1,56 & numeral & 256676856 & 2,08 \\
\hline possessive adjective & 15045 & 1,39 & possessive adjective & 165210187 & 1,34 \\
\hline verb past simple & 11258 & 1,04 & verb past simple & 64623115 & 0,52 \\
\hline indefinite pronoun & 8542 & 0,79 & indefinite pronoun & 76526069 & 0,62 \\
\hline verb present participle & 5790 & 0,54 & verb present participle & 53962889 & 0,44 \\
\hline verb future & 3576 & 0,33 & verb future & 58690287 & 0,47 \\
\hline verb conditional & 2655 & 0,25 & verb conditional & 34393352 & 0,28 \\
\hline verb subjonctive present & 1590 & 0,15 & verb subjonctive present & 19351932 & 0,16 \\
\hline
\end{tabular}




\section{Revista Científica ECOCIENCIA}

\begin{tabular}{|c|c|c|c|c|c|}
\hline verb subjunctive imperfect & 1220 & 0,11 & verb subjunctive imperfect & 16056120 & 0,13 \\
\hline abbreviation & 1181 & 0,11 & abbreviation & 48105451 & 0,39 \\
\hline interjection & 463 & 0,04 & interjection & 8759991 & 0,07 \\
\hline pronoun & 176 & 0,02 & pronoun & 2100832 & 0,02 \\
\hline possessive pronoun & 127 & 0,01 & possessive pronoun & 1322824 & 0,01 \\
\hline symbol & 42 & 0,00 & symbol & 12570641 & 0,10 \\
\hline verb imperative & 13 & 0,00 & verb imperative & 438367 & 0,00 \\
\hline Total & 1078496 & 100 & Total & 12369868562 & 100 \\
\hline
\end{tabular}

Fuente: obtenido del programa en línea SketchEngine.com

Es interesante constatar que en el género textual "anécdota" se observa porcentajes relativamente parecidos a los porcentajes representativos del corpus general a pesar de conservar proporciones que traducen su dimensión narrativa. El porcentaje de los verbos en presente es inferior de - 0,57 puntos y el porcentaje de los verbos en pasado es superior de $+0,92$ puntos por el imperfecto, y de $+0,52$ por el pretérito. La diferencia de porcentaje entre los pronombres personales $(+1,2)$ comparado con el porcentaje de los nombres propios $(-1,43)$ es en parte debido al carácter anafórico del pronombre personal en una narración reemplaza regularmente los personajes de la historia. Ninguno de los datos obtenidos permite una clasificación genérica de la anécdota en un dominio específico - que sea turístico o no. Solo la enunciación dentro de un contexto turístico autoriza una ubicación precisa de la anécdota en el discurso profesional del turismo según las categorías introducidas por Mapelli \& Piccioni (2011).

Esta categorización siendo relacionada con el contexto, se ilustra con el análisis de las palabras claves y de las expresiones propias en el cuadro 2. La obtención de estas palabras claves o, más explícitamente, n-grams $\mathrm{N}$ propios, se calcula según una tasa de frecuencias normalizadas extraídas de los dos corpus añadiendo una constancia para evitar la división por cero (caso de una palabra presente únicamente en uno de los dos corpus) y para favorecer las palabras cuya frecuencia es significativa (Kilgarriff, 2012, pp. 4-6); o sea (f $f_{\text {focus }}$ $+n) /\left(f_{\text {ref }}+n\right)$ con $f_{\text {focus }}$ como frecuencia normalizada de la palabra en el corpus estudiado - 
"anécdota", $f_{\text {ref }}$ frecuencia normalizada de la palabra en el corpus de referencia y $n$ es igual a 100. Los resultados de las 52 palabras claves y de las 10 expresiones propias las más representativas son expuestas en la Tabla 2 seguidas de, entre paréntesis, el valor de la tasa y de la frecuencia de la palabra en el corpus estudiado.

Tabla 2. Palabras claves y expresiones propias constitutivas del corpus «anécdota» en referencia al corpus «frTenTen12».

\begin{tabular}{|c|c|c|c|}
\hline \multicolumn{4}{|c|}{ Palabras claves } \\
\hline roi $(4,9$ / 908) & étaient (2,7 / 1098) & foule $(2,3$ / 213) & autrefois $(2,1 / 165)$ \\
\hline fut $(4,2$ / 1513) & noël (2,6 / 307) & fût $(2,3$ / 186) & église (2,1 / 291) \\
\hline siècle $(4,1$ / 899) & cent $(2,6$ / 299) & pain (2,3 / 216) & charles $(2,1 / 194)$ \\
\hline Iouis (3,9 / 502) & avaient (2,5 / 698) & lettres (2,3 / 252) & molière (2,0 / 122) \\
\hline eût (3,0 / 263) & mille $(2,4$ / 277) & rois $(2,3$ / 175) & $\operatorname{cour}(2,0$ / 286) \\
\hline furent $(3,0$ / 384) & jusqu’ (2,4 / 805) & peuple (2,2 / 402) & siècles (2,0 / 179) \\
\hline fête (3,0 / 427) & $\operatorname{vin}(2,4$ / 271) & $\operatorname{xiv}(2,2$ / 143) & aussitôt (2,0 / 172) \\
\hline henri (2,9 / 281) & cœur (2,4 / 154) & xixe $(2,2$ / 149) & époque (2,0 / 397) \\
\hline eut $(2,9$ / 390) & chroniqueur (2,4 / 160) & dont $(2,1 / 1642)$ & bourgeois $(2,0$ / 131) \\
\hline paris $(2,8$ / 904) & duc $(2,4$ / 195) & heures $(2,1 / 596)$ & suivant (2,0 / 282) \\
\hline fit $(2,8$ / 508) & francs $(2,4$ / 216) & c'est-à-dire (2,1 / 202) & quelquefois $(2,0$ / 131) \\
\hline$q u^{\prime}(2,8$ / 7653) & honneur (2,4 / 288) & fleurs (2,1 / 209) & ville $(2,0$ / 714) \\
\hline usage $(2,7$ / 395) & coutumes (2,3 / 169) & napoléon (2,1 / 140) & fêtes (2,0 / 165) \\
\hline \multicolumn{4}{|c|}{ Expression } \\
\hline jeunes gens $(1,9)$ & heures du soir $(1,5)$ & messe de minuit $(1,3)$ & légion d'honneur $(1,3)$ \\
\hline heures du matin $(1,6)$ & grand nombre $(1,4)$ & neuf heures $(1,3)$ & \\
\hline point de vue $(1,6)$ & jeunes filles $(1,3)$ & même temps $(1,3)$ & \\
\hline
\end{tabular}

Fuente: obtenido del programa en línea SketchEngine.com 
En el contexto de la visita guiada aparecen palabras relacionada con la narración histórica: "roi(s), Louis, Henri, fête(s), Paris, vin, Duc, coutume, peuple, Napoléon, autrefois, église, Charles, Molière, cour, siècle, époque, bourgeois" ; con este género: "fut, eût, furent, eut, fit, étaient, avaient, fût". En cuanto al sintagma "légion

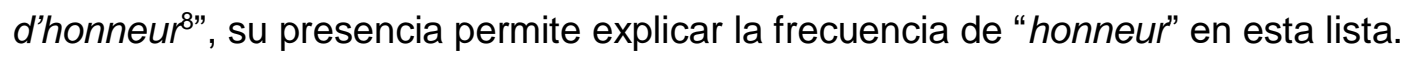

Cinco expresiones propias sobre diez presentan una dimensión temporal, referencia necesaria para ubicar el contexto histórico y los hechos relacionados. Las expresiones propias "jeunes gens, jeunes filles, grand nombre" suenan un poco anticuadas, pero refuerzan el carácter enfático del estilo narrativo de la anécdota. Una palabra clave parece extraña en la lista: "chroniqueur"; pero con la ayuda de la concordancias es fácil notar que esta palabra es recurrente en las citaciones de las fuentes de las anécdotas. Es entonces lógico que aparezca en las palabras claves con la función de identificar el supuesto autor de la anécdota. Las concordancias realizadas con estas palabras claves confirman la posición de las anécdotas en el contexto histórico narrativo en adecuación con la pertenencia al macro-género textual de la "guía" y a la familia de género textual "institucional" según la taxonomía de Mapelli et Piccioni (2011):

... les chanceliers, les princes de Condé, les $<$ rois $>$ de Navarre ; et je vous ai en tête, petit

... mars, en chacun 3 livres d'amende envers le $<$ roi > ; un sixième, non comparant, est condamné

... là le vrai but de ces tables d'hôte qui < furent $>$ fermées après le décret de LouisPhilippe

... qui, en dehors de tout " don " personnel, < furent $>$ jadis glorieuses et qui sont, aujourd'hui

... permettant de s'agripper à la neige. Le < chroniqueur > scientifique Paul Perrin s'arrête sur cette

\footnotetext{
6 “rey(es), Luis, Henri, fiesta(s), Paris, vino, Duque, costumbre, pueblo, Napoléon, antaño, iglesia, Carlos, Molière, corte, siglo, época, burgués"

7 "fue, tuviese (tuviera), fueron, tuvo, hizo, estaban, tenían, fuese (fuera)"

8 "Legión de honor"

9 "cronista"
}

ISSN: 1390-9320, Vol. 5, No.5, octubre 2017 
... mieux, je veux dire plus drôle, poursuit le < chroniqueur >. En 1691, l'argentier de Louis XIV s'avisa

\section{ANÁLISIS DE LOS RESULTADOS Y DISCUSIÓN}

Uno de los resultados, el más destacado, de este análisis es la ausencia de evidencias en favor de la existencia de un sub-sistema lingüístico asociado a una especialidad o a un dominio específico. Aún si la anécdota no es un género textual exclusivamente del dominio del turismo, cuando se enmarca en este discurso situado, el estudio revela únicamente sus características narrativas y discursivas propias. Estos elementos son evidenciados por la frecuencia de las categorías gramaticales utilizadas dentro de un corpus cuya densidad permite garantizar la representatividad y especificidad del texto estudiado, lo que no permite un estudio manual que no puede interrogar una cantidad de datos tan importante. Algunas palabras características de la temática y del contexto traducen la pertenencia de la anécdota al dominio del turismo, pero no representan una terminología propia al turismo ya que no son ausentas del corpus "común" representativo del lenguaje generalmente considerado como ordinario, confirmando la idea de una especialización del discurso por su situación comunicativa y no por la existencia de un sub-sistema lingüístico.

Esta realidad permite imaginar una contextualización del proceso de enseñanzaaprendizaje de un idioma extranjero en situaciones profesionales desde los primeros niveles, entrando en adecuación con el "giro praxeológico" que vuelve a centrar el proceso sobre la interacción social apoyándose en el constructivismo social definido por Vygotsky. El desarrollo de esta conceptualización interactiva inspiró expertos como Charaudeau quien define la competencia discursiva como la capacidad de activar "los procesos de puesta en escena discursiva considerando las obligaciones del marco situacional [que] son de tres niveles: enunciativo, organizativo y semántico ${ }^{10 "}(2000$, p.38), es decir relativos a la situación de enunciación, a la organización del discurso, y a los saberes compartidos.

\footnotetext{
${ }^{10}$ les procédés de mise en scène discursive qui font écho aux contraintes du cadre situationnel [qui] sont de trois ordres : énonciatif, énoncif et sémantique
} 
El principal interés de construir contenidos para el proceso de enseñanza-aprendizaje siguiendo estos tres niveles es disponer de una base empírica más representativa de la realidad profesional que la creación de contenido de manera introspectiva. En efecto, el corpus provee ejemplos de lo que el locutor piensa ser una producción correcta de su lenguaje (McEnery, Xiao \& Tono, 2006, p. 6); y el hecho de considerar los resultados del análisis de un discurso producido en situaciones de comunicación profesional para proponer contenidos pragmáticos con el afán de desarrollar las competencias lingüísticas, responde a las exigencias de la segunda hipótesis de Krashen (1977) sobre el aprendizaje de las lenguas extranjeras. Son contenidos accesibles y comprensibles por los estudiantes por el hecho de ser compilados a partir del dominio que eligieron estudiar, son altamente significativos y son modelos para producir textos y discursos adaptados a las futuras situaciones profesionales que enfrentaran (Bronckart, 2011, p. 44)

\section{CONCLUSIONES}

La lingüística de corpus permite proponer una descripción detallada del carácter pragmático de los discursos producidos en las situaciones de comunicación que el experto habrá elegido estudiar. La integración de esta información al nivel de la selección de los contenidos del proceso de enseñanza-aprendizaje permite estructurar el desarrollo de las competencias comunicativas requeridas con base en los materiales auténticos, en este caso relacionados al dominio del turismo. Aún si la lógica fuese que estos contenidos sean presentados de manera interaccionales relativamente a las situaciones de comunicación, varias investigaciones didácticas pueden ser propuestas para determinar las metodologías más adaptadas a esta integración.

\section{REFERENCIAS BIBLIOGRÁFICAS}

Bronckart, J-P. (2011). La formation aux compétences langagières : pour un réexamen des rapports entre langues et discours. Bulletin VALS-ASLA, volumen V. (93) 2011, 27-46.

ISSN: 1390-9320, Vol. 5, No.5, octubre 2017 
Calvi, M. V. (2010). Los géneros discursivos en la lengua del turismo: una propuesta de clasificación. Ibérica, volumen V. (19) 2010, 9-31.

Charaudeau, P. (2000). De la compétence sociale de communication aux compétences de discours. Didactique des langues romanes. Le développement des compétences chez l'apprenant. Bruxelles :De Boeck-Duculot. 34-43.

Conseil de l'Europe. (2001). Cadre Européen Commun de Référence pour le Langues : apprendre, enseigner, évaluer. Paris : Les éditions Didier.

Corbeau, S.; Dubois, C.; Penfomis, J-L. (2004). Tourisme.com. Paris : Clé international. ISBN : 978-2-09-033174-5.

Corbeau, S.; Dubois, C.; Penfomis, J-L.; Semichon, L. (2013). Hôtellerie-restauration.com. Paris : Clé international. ISBN : 978-2-09-038046-0.

Gautier, L. (2014). Des langues de spécialité à la communication spécialisé : un nouveau paradigme de recherche à l'intersection entre sciences du langage, info-com et sciences cognitives ? Etudes Interdisciplinaires en Francophonie. Sciences humaines, volumen V. (1) 2014, 225-245.

Girardet, J.; Pecheur, J. (2006). Campus 1. Paris : Clé international. ISBN : 978-2-09033308-4.

Germain, C. (1993). Evolution de l'enseignement des langues : 5000 ans d'histoire. Paris : CLé international.

Gossman, L. (2003). Anecdote And History. History and Theory, volumen V. (42) 2003, $143-168$.

Gunnarsson, B-L. (2009). Professional Discourse. London and New York : Continuum International Publishnig Group.

Hymes, D. H. (1992). The concept of communicative competence revisited. Thirty Years of Linguistic Evolution. Amsterdam/Philadelphia : Benjamins. 31-58.

Kilgarriff, A. Getting to know your corpus. Recuperado el 28 de mayo 2014 https://www.sketchengine.co.uk/xdocumentation/attachment/wiki/AK/Papers/Kilgarr iff TSD2012.pdf

Krashen, S. (1977). Some issues relating to the monitor model. Teaching and learning English as a Second Language: Trends in Research and Practice: On TESOL '77: Selected Papers from the Eleventh Annual Convention of Teachers of English to Speakers of Other Language. Washington, DC: Teachers of English to Speakers of Other Languages. 144-158. 
Lavric, E. (2013). Wie Europas Winzer/innen Fremdsprachen lernen - Präsentation des EU-Projekts 'VinoLingua'. Fachsprache(n) in der Romania,Entwicklung, verwendung, Übersetzung, Wienen. Berlin: Frank und Timme. 309-33.

Lerat, P. (1995). Les langues spécialisées. Paris : Presse Universitaires de France.

Mapelli, G.; Piccioni, S. (2011). Taxonomía de los textos turísticos: factores lingüísticos y factores contextuales. La lengua del turismo géneros discursivos y terminología. Bern: Peter Lang.

McEnery, T.; Xiao, R.; Tono, Y. (2006). Corpus-Based Language Studies. London, New York : Routledge.

Petit, M. (s.f.). Le discours spécialisé et le spécialisé du discours : repère pour l'analyse du discours en anglais de spécialité. Recuperado el 28 de mayo 2014. http://erea.revues.org/1400

Richard, J. C. (2001). Curriculum Development in Language Teaching. $1^{\text {St }}$ ed. Cambridge : Cambridge University Press. 